\title{
Das kutane Lymphom beim Hund - Verwechslungsgefahr!
}

Tanja Töpfer

\section{Das kutane Lymphom des Hundes ist sehr variabel in Bezug auf das klinische Erscheinungsbild, z. B. Verteilungsmuster, Pruritus und Prognose. Aufgrund dieser Heterogenität kann es zu Verwechslungen mit anderen Hauterkrankungen kommen wie der atopischen Dermatitis oder der Sarkoptesräude.}

\section{Einleitung}

Das kutane Lymphom beim Hund ist mit einer Prävalenz von ca. $1 \%$ eine seltene neoplastische Hauterkrankung. Auch unter den kaninen Lymphomen tritt es mit 3-8\% deutlich seltener auf als die multizentrische Form der Krankheit [1]. Betroffen sind vor allem ältere Tiere ohne besondere Geschlechtsprädisposition. Allerdings besteht eine Rasseprädisposition für den Cocker Spaniel und Boxer [2].

\section{Ätiologie}

Die genaue Ätiologie des kutanen Lymphoms beim Hund ist unklar. Es wird diskutiert, ob eine vorhergehende chronische Entzündung sowohl beim Menschen als auch bei Hunden zu der Entstehung der Erkrankung beitragen kann. In einigen Studien konnte ein Zusammenhang zwischen dem Vorliegen einer atopischen Dermatitis und der Entwicklung eines kutanen Lymphoms festgestellt werden. In einer amerikanischen Studie wurden 19 Hunde mit kutanem Lymphom untersucht, wobei 5 Hunde im Vorfeld eine allergische Hauterkrankung aufwiesen [3]. Bezogen auf die Gesamtpopulation der Klinik hatten die Tiere mit atopischer Dermatitis ein 12-fach höheres Risiko für die Entstehung eines Lymphoms. Die Erkrankungen weisen ähnliche Lymphozytenphänotypen, Zytokinspiegel und einen erhöhten lgE-Antikörperspiegel auf [3]. Man geht davon aus, dass es durch die chronische Aktivierung der Lymphozyten schließlich zu einer Proliferation und neoplastischen Entartung kommt.

Beim epitheliotropen Lymphom kommt die Affinität der T-Lymphozyten für die Epidermis und adnexalen Strukturen (Haarfollikel und Drüsen) vor allem durch die Expression von sogenannten Skin-Homing-Rezeptoren, z. B. CCChemokin-Rezeptor-4, zustande [4]. Diese sorgen für die Bindung an die Keratinozyten und Langerhans-Zellen.

\section{Klinisches Bild}

\section{Merke}

Die Ausprägung der Hautläsionen, die Verteilung und das Vorkommen von Pruritus sind sehr variabel.

Deswegen kann die Erkrankung mit zahlreichen anderen Dermatosen oder Endokrinopathien verwechselt werden. Häufig werden die Tiere bereits mit einer chronischen Symptomatik und zahlreichen Vorbehandlungen präsentiert. Die Zeit zwischen den ersten Symptomen und der Diagnosestellung ist häufig sehr lang (durchschnittlich ca. 5 Monate) [1]. Ca. 40\% der Tiere zeigen Pruritus in unterschiedlichen Schweregraden [6]. Das Fehlen von Juckreiz sollte daher nicht zum Ausschluss der Differenzialdiagnose kutanes Lymphom führen.

In einer Studie ergaben sich bei 30 Hunden folgende Verteilungen der Läsionen [6]:

- Rumpf: 83,3\%

- Kopf: $63 \%$

- Nase: $43 \%$

- Ballen: 26,6\%

Diese 30 Hunde wiesen folgende Primär- und Sekundärläsionen der Haut auf [6]:

- Erythem: 86,6\%

- Plaque: $73,3 \%$

- Erosion: $60 \%$

- Schuppen: $60 \%$

- Knoten: 53,3\%

- Hypopigmentation: $50 \%$

- Krusten: $46,6 \%$

- Alopezie: $33,3 \%$ 
- Tab. 1 Pathohistologische Unterscheidung der Subformen des kutanen Lymphoms beim Hund [2].

\begin{tabular}{|l|l|}
\hline Subform & Befunde \\
\hline $\begin{array}{l}\text { Mycosis } \\
\text { fungoides }\end{array}$ & $\begin{array}{l}\text { diffuse Verteilung der neoplastischen Lymphozyten oder } \\
\text { Bildung von intraepidermalen Pusteln (Pautrier's Mikro- } \\
\text { abszesse) }\end{array}$ \\
\hline $\begin{array}{l}\text { pagetoide } \\
\text { Retikulose }\end{array}$ & $\begin{array}{l}\text { strikt intraepitheliale und periadnexale Anreicherung } \\
\text { der neoplastischen Zellen }\end{array}$ \\
\hline Sézary- & $\begin{array}{l}\text { Nachweis von Sézary-Zellen mit charakteristischem } \\
\text { gehirnwindungsartigen Zellkern in der Haut, aber } \\
\text { gleichzeitig auch im peripheren Blut und Lymphknoten; } \\
\text { Differenzialdiagnose: lymphozytäre Leukämie }\end{array}$ \\
\hline
\end{tabular}

\section{Einteilung}

Es werden 2 Formen der Erkrankung anhand der Verteilung der neoplastischen Lymphozyten beschrieben:

- epitheliotropes Lymphom: Infiltration neoplastischer T-Lymphozyten mit Tropismus für die Epidermis und adnexalen Strukturen

- nicht epitheliotropes Lymphom: primär mit Ursprung der neoplastischen Zellen in der Dermis oder sekundär im Zusammenhang mit einem multizentrischen Lymphom

Angelehnt an die Humanmedizin werden beim epitheliotropen Lymphom außerdem 3 weitere Subformen unterschieden:

- Mycosis fungoides

- pagetoide Retikulose

- Sézary-Syndrom

Diese Unterscheidung kann nicht durch das klinische Bild des Patienten getroffen werden, sondern kann nur anhand der histopathologischen Befunde erfolgen ( $\triangleright$ Tab. 1). Die Mycosis fungoides tritt deutlich häufiger auf als die beiden anderen Formen. Für die pagetoide Retikulose und das Sézary-Syndrom gibt es in der Veterinärmedizin nur wenige Fallberichte [5].

\section{Klassifikation des epitheliotropen Lymphoms}

Es empfiehlt sich die Einteilung der kutanen Läsionen nach folgender Klassifikation [2]:

- exfoliative Erythrodermie

- Patch-, Plaque- oder Knotenform

- mukokutane Form

- orale Form

Diese Formen können sowohl gleichzeitig als auch losgelöst voneinander klinisch in Erscheinung treten. Sie müssen nicht mit der Dauer oder Prognose der Erkrankung korrelieren.

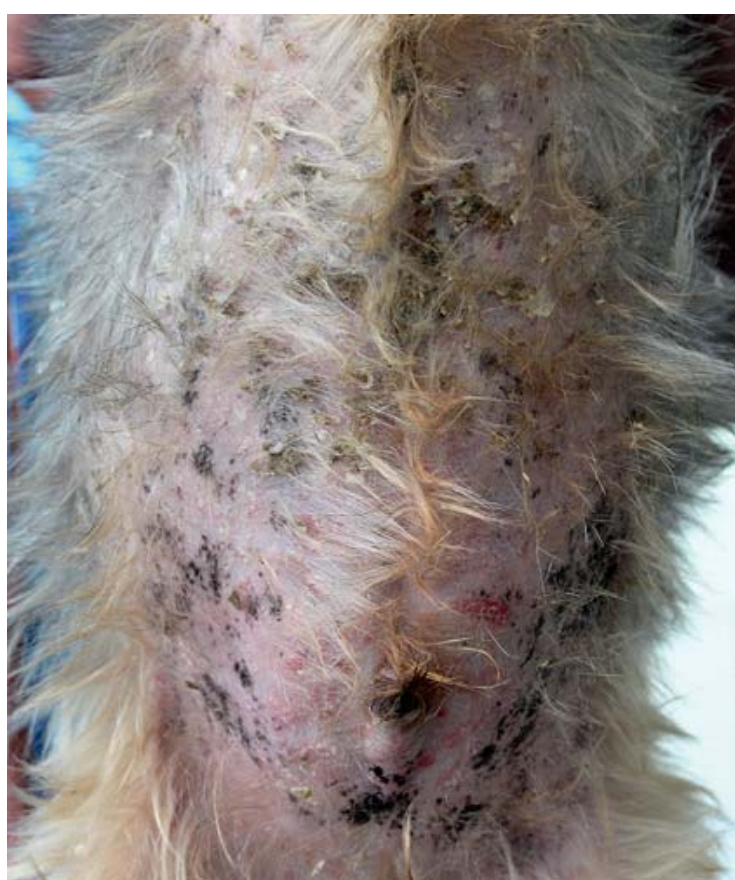

- Abb. 1 Yorkshire Terrier im Stadium der exfoliativen Erythrodermie durch ein kutanes Lymphom mit einer vermehrten Schuppenbildung und Rötung am ventralen Abdomen. @ Universität Leipzig, Klinik für Kleintiere

\section{Exfoliative Erythrodermie}

Eine Rötung der Haut sowie die vermehrte Schuppenbildung können an verschiedenen Lokalisationen des Körpers auftreten ( $\bullet$ Abb. 1). Vor allem der Rumpf und der Kopf sind gehäuft betroffen. Da die Ausprägung von Juckreiz variabel ist, kann es zu Sekundärläsionen wie Erosionen oder Ulzerationen kommen. Dieses Stadium kann für den Besitzer ein vergleichsweise harmloses Aussehen annehmen, sodass die Tiere in einigen Fällen nicht in der Sprechstunde vorgestellt werden. Auch für den behandelnden Tierarzt können diese Hautveränderungen eine Herausforderung darstellen, da es zu einer Verwechslung mit Allergien oder der Sarkoptesräude kommen kann. Dies geschieht vor allem dann, wenn Lokalisationen mit Juckreiz betroffen sind, die sonst üblicherweise bei diesen Erkrankungen vorkommen. Bei älteren Hunden mit nicht juckenden Schuppenbildungen am Rumpf kann auch fälschlicherweise eine Endokrinopathie, z. B. Hypothyreose, als Ursache vermutet werden.

\section{Patch-, Plaque- oder Knotenform}

Hierbei handelt es sich um das Stadium, in dem die Hunde am häufigsten beim Tierarzt vorgestellt werden, da vor allem bei knotigen Veränderungen bereits makroskopisch der Verdacht auf eine Tumorerkrankung gestellt werden kann. Die Tiere zeigen initial häufig nur 1 oder 2 gut umschriebene Patches ( $\boldsymbol{A} \mathbf{A b b} . \mathbf{2}$ ) oder Plaques ( Abb. 3), die schuppig, alopezisch oder krustig verändert sein können. Eine schrittweise Vergrößerung oder 


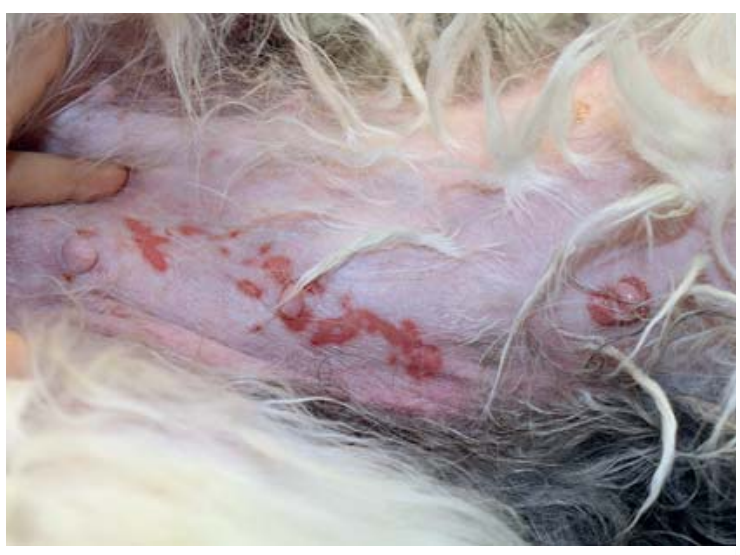

- Abb. 2 Bobtail mit kutanem Lymphom im Patchstadium mit fleckigen Rötungen am ventralen Abdomen. (c) Universität Leipzig, Klinik für Kleintiere

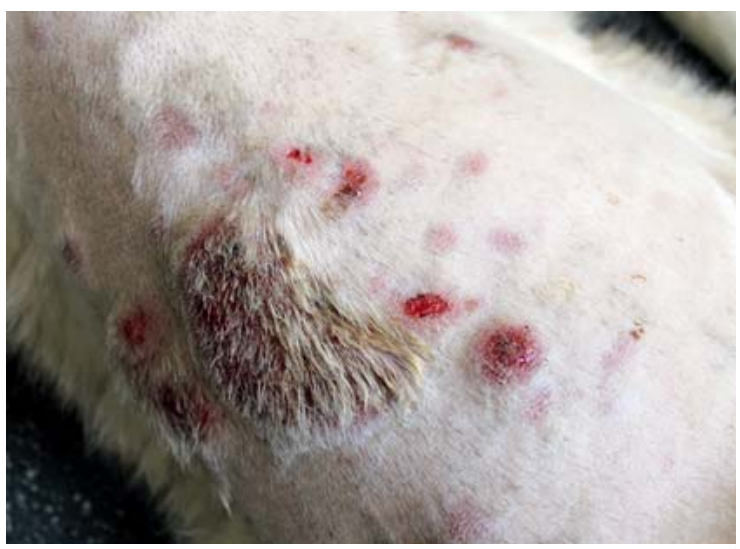

- Abb. 3 Saarloos Wolfshund mit der Diagnose eines kutanen Lymphoms. Zu sehen sind plaqueartige Hautveränderungen am Rücken. @ Universität Leipzig, Klinik für Kleintiere

die Entwicklung zu einem Knoten ist möglich. Auch hier sind die Lokalisation und das Vorhandensein von Juckreiz variabel.

\section{Mukokutane Form}

Bei dieser Form sind vor allem die Lippen ( $\bullet$ Abb. 4), die Nase und die Augenlider ( $\bullet$ Abb. $\mathbf{5}$ ) von einer vermehrten Rötung, Hypopigmentation und Erosion betroffen.

\section{Orale Form}

Circa $50 \%$ der Hunde weisen mukosale Veränderungen auf [6]. In manchen Fällen fehlen kutane Läsionen vollständig und Rötungen und Ulzerationen an Zunge, Gingiva ( $\bullet$ Abb. 6 ) oder Gaumen sind die einzigen Auffälligkeiten am Tier. Vor allem in diesen Fällen ist die Verwechslungsgefahr für den Tierarzt besonders hoch, da es fälschlicherweise zur Diagnose einer Gingivitis oder Autoimmunerkrankung wie dem Pemphigus foliaceus kommen kann. 


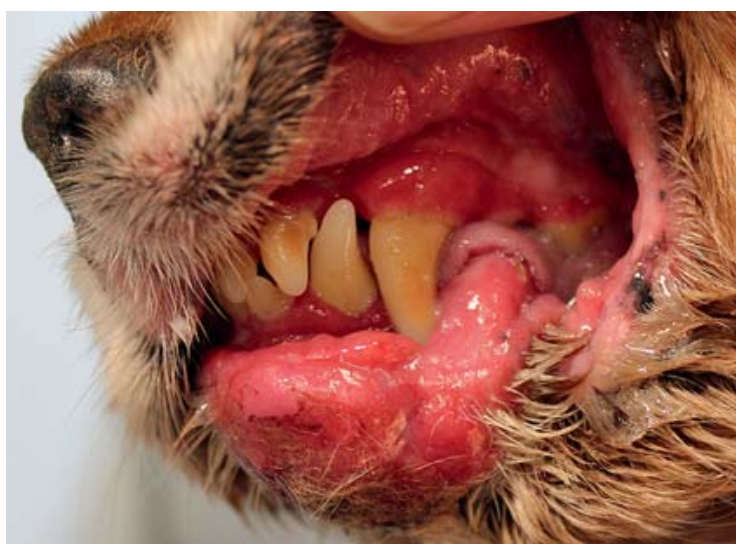

- Abb. 4 Cocker Spaniel mit der mukokutanen Form des kutanen Lymphoms. Es ist zu einer Rötung und Umfangsvermehrung an den Lefzen gekommen. ( U Universität Leipzig, Klinik für Kleintiere

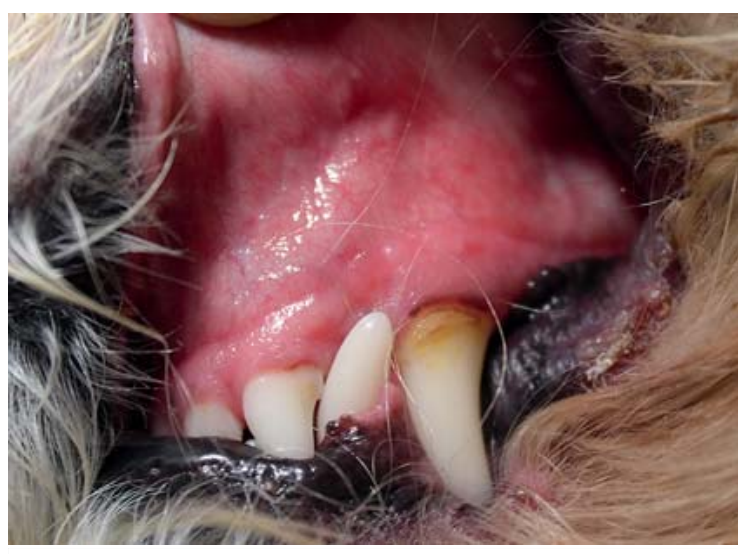

- Abb. 6 Yorkshire Terrier mit Rötungen der Maulschleimhaut, bedingt durch ein orales kutanes Lymphom. (c) Universität Leipzig, Klinik für Kleintiere

\section{Diagnostik}

\section{WARNHINWEISE}

Folgende Punkte sollten als Warnhinweise für das Vorkommen eines kutanen Lymphoms gewertet werden:

- Ballen- oder Nasenspiegelveränderungen

- gleichzeitiges Auftreten von Läsionen an der Haut und Schleimhaut

- plötzliche Verschlechterung eines gut eingestellten Allergikers ohne offensichtliche Ursache

- gleichzeitige Hautveränderungen und generalisierte Lymphadenomegalie

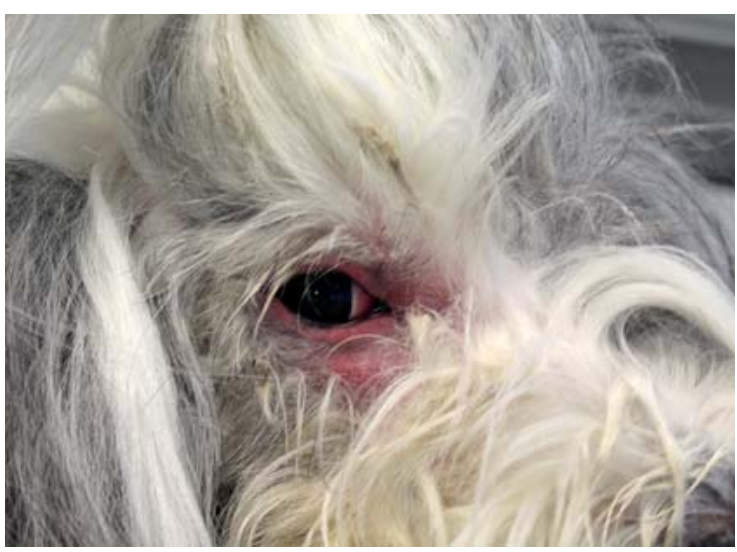

- Abb. 5 Bobtail mit einer Rötung der Augenlider, ausgelöst durch die mukokutane Form des kutanen Lymphoms. @ Universität Leipzig, Klinik für Kleintiere

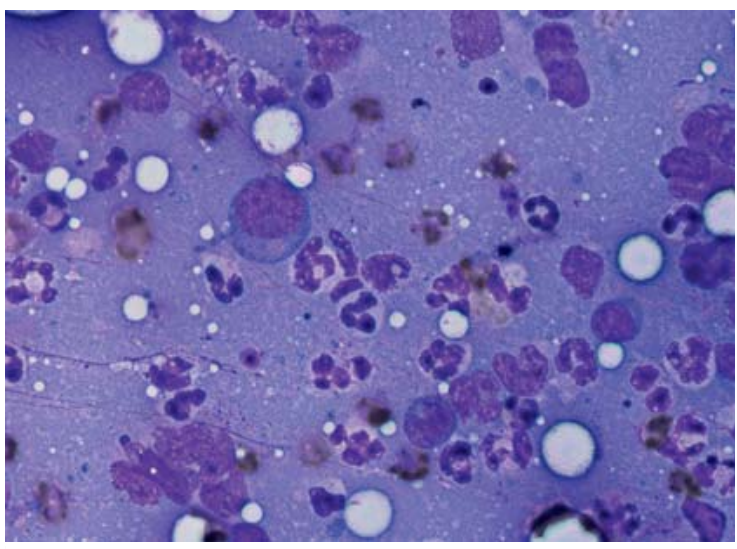

- Abb. 7 Abklatschpräparat der Haut. Zytologisch sind eine Rundzellpopulation und zahlreiche neutrophile Granulozyten sichtbar. @ Universität Leipzig, Klinik für Kleintiere

\section{Zytologie}

Neben dem klinischen Bild kann auch die Anfertigung einer zytologischen Untersuchung hilfreich für die Aufstellung einer Verdachtsdiagnose sein. Hierbei kann bei nässenden Läsionen eine Abklatschzytologie angefertigt werden.

\section{Merke}

Sind vor allem knotige Hautveränderungen ausgebildet, empfiehlt sich die Feinnadelaspiration.

Die Interpretation der Präparate kann insbesondere bei purulenten Läsionen aufgrund der Überlagerung mit neutrophilen Granulozyten erschwert werden. Bei den neoplastischen Zellen handelt es sich um lymphatische Rundzellen mit breitem Zytoplasmasaum und großem Nukleus ( $\triangleright$ Abb. 7). 


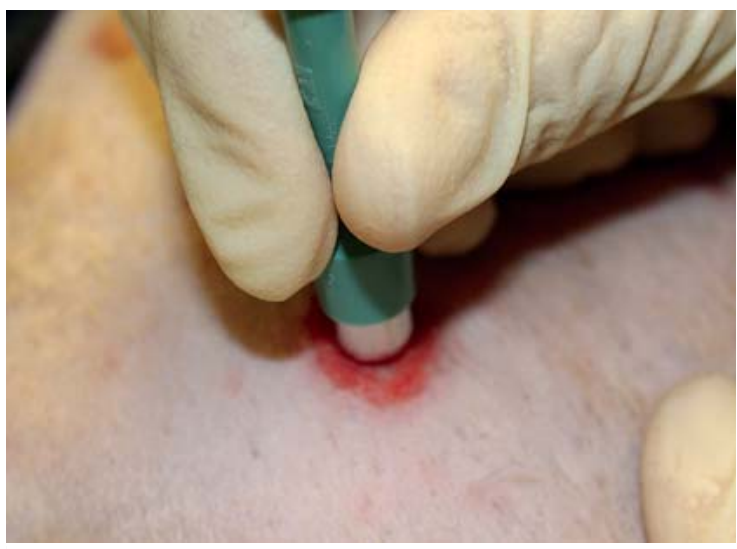

- Abb. 8 Entnahme einer Hautbiopsie mit einer 8 mm Stanze zentral in der Hautläsion. (c) Universität Leipzig, Klinik für Kleintiere

\section{Histopathologische Untersuchung}

\section{Merke}

Die sichere Diagnosestellung gelingt nur durch die Entnahme von Hautbiopsien mit nachfolgender histopathologischer Untersuchung.

Nur hier kann der Epitheliotropismus der Zellen dargestellt und eine Unterscheidung der Subformen vor- genommen werden ( $\triangleright$ Tab. 1). Dabei sollten die Stanzbiopsien möglichst zentral aus den repräsentativsten Primärläsionen wie Papeln, Knoten oder schuppigen Hautbereichen genommen werden ( $\triangleright$ Abb. 8). Vermieden werden sollte die Beprobung stark infizierter oder ulzerierter Hautstellen.

Für den Pathologen kann sich die Unterscheidung zu anderen Rundzelltumoren der Haut, z. B. undifferenzierten Mastzelltumoren oder histiozytären Neoplasien, schwierig gestalten. Durch immunhistochemische Untersuchungen kann das Vorliegen von $\mathrm{CD}^{+}$-T-Lymphozyten bestätigt werden [7]. Zusätzlich kann durch eine PCR-Untersuchung auf den T-Zell-Rezeptor die Unterscheidung zu lymphozytären Dermatitiden gelingen, welche beispielsweise beim Lupus erythematodes oder dem Erythema multiforme auftreten können [8,9].

\section{Therapie}

\section{Merke}

Bei der Auswahl der optimalen Therapie muss unterschieden werden, ob es sich um eine solitäre Läsion oder um eine generalisierte Erkrankung handelt.

Ist nur eine Lokalisation betroffen, sollte eine chirurgische Exzision erwogen werden. Dieser Eingriff kann bei ca. $50 \%$ der Patienten sogar kurativ sein [10]. Alternativ

- Tab. 2 Systemische Behandlungsoptionen (inkl. Dosierungen und Nebenwirkungen) beim kutanen Lymphom des Hundes.

\begin{tabular}{|c|c|c|c|c|c|}
\hline Stoffklasse & Wirkstoff & Dosierung & $\begin{array}{l}\text { Wirkmecha- } \\
\text { nismus }\end{array}$ & Ansprechrate & Nebenwirkungen \\
\hline \multirow[t]{2}{*}{ Zytostatika } & $\begin{array}{l}\text { Lomustin (Chlorethyl- } \\
\text { Cyclohexyl-Nitroso-Urea, } \\
\text { CCNU) }\end{array}$ & $\begin{array}{l}60-70 \mathrm{mg} / \\
\mathrm{m}^{2} \text { p.o. alle } \\
3 \text { Wochen }\end{array}$ & $\begin{array}{l}\text { alkylierender } \\
\text { Effekt auf die } \\
\text { DNA }\end{array}$ & $\begin{array}{l}\text {. 78-82\%iges An- } \\
\text { sprechen für me- } \\
\text { dian 94-106 Tage } \\
\text { " mittlere Über- } \\
\text { lebenszeit } 6 \text { Mo- } \\
\text { nate }[14,15]\end{array}$ & $\begin{array}{l}\text { - gastrointestinale Beschwerden } \\
\text { [13] } \\
\text { - Leukopenie [13] } \\
\text { - Thrombozytopenie [13] } \\
\text { - Hepatotoxizität [13] }\end{array}$ \\
\hline & $\begin{array}{l}\text { multimodale intravenöse } \\
\text { Protokolle, z. B. Doxoru- } \\
\text { bicin, L-Asparaginase, } \\
\text { Vincristin, Methotrexat, } \\
\text { Mitoxantrone }\end{array}$ & variabel & variabel & $\begin{array}{l}\text { - 15-44\%iges } \\
\text { Ansprechen } \\
\text { - mittlere Über- } \\
\text { lebenszeit } \\
\text { 2-6 Monate }\end{array}$ & $\begin{array}{l}\text { - gastrointestinale Beschwerden } \\
\text { - Leukopenie } \\
\text { - Thrombozytopenie }\end{array}$ \\
\hline $\begin{array}{l}\text { Tyrosinkinase- } \\
\text { Inhibitoren }\end{array}$ & Masitinib (Masivet ${ }^{\circledR}$ ) & $\begin{array}{l}12,5 \mathrm{mg} / \mathrm{kg} / \\
\text { Tag p. o. }\end{array}$ & $\begin{array}{l}\text { antiprolifera- } \\
\text { tiver Effekt } \\
\text { auf lymphoide } \\
\text { Zellen }\end{array}$ & $\begin{array}{l}\text { 70\%iges Ansprechen } \\
\text { für median } 85 \text { Tage } \\
\text { [16] }\end{array}$ & $\begin{array}{l}\text { - Proteinurie } \\
\text { - hämolytische Anämie } \\
\text { - Hepatotoxizität } \\
\text { - Neutropenie }\end{array}$ \\
\hline $\begin{array}{l}\text { synthetische } \\
\text { Retinoide (Vita- } \\
\text { min-A-Analoga) }\end{array}$ & Isotretinoin, Etretinat & $\begin{array}{l}3 \mathrm{mg} / \mathrm{kg} / \\
\text { Tag p.o. }\end{array}$ & $\begin{array}{l}\text { antiprolifera- } \\
\text { tiver Effekt }\end{array}$ & $\begin{array}{l}\text { Ansprechen bei } \\
6 \text { von } 14 \text { Hunden für } \\
5-17 \text { Monate }[18]\end{array}$ & Hepatotoxizität \\
\hline $\begin{array}{l}\text { ungesättigte } \\
\text { Fettsäuren }\end{array}$ & $\begin{array}{l}\text { Omega-6-Fettsäuren } \\
\text { (Linolensäure, z. B. } \\
\text { Nachtkerzenöl) }\end{array}$ & $\begin{array}{l}3 \mathrm{ml} / \mathrm{kg} / \mathrm{Tag} \\
\text { p.o. }\end{array}$ & $\begin{array}{l}\text { antioxidativ, } \\
\text { antiinflamma- } \\
\text { torisch }\end{array}$ & $\begin{array}{l}\text { Ansprechen bei } 6 \text { von } \\
8 \text { Hunden [17] }\end{array}$ & $\begin{array}{l}\text { selten gastrointestinale } \\
\text { Beschwerden }\end{array}$ \\
\hline Kortikosteroide & Prednisolon & $\begin{array}{l}\text { 0,5-2 mg/ } \\
\text { kg/Tag p. o. }\end{array}$ & $\begin{array}{l}\text { Hemmung der } \\
\text { Angiogenese }\end{array}$ & $\begin{array}{l}\text { mittlere Überlebens- } \\
\text { zeit ca. } 60 \text { Tage [10] }\end{array}$ & $\begin{array}{l}\text { - Polyurie/Polydipsie } \\
\text { - Polyphagie } \\
\text { - gastrointestinale Beschwerden }\end{array}$ \\
\hline
\end{tabular}


- Tab. 3 Günstige und ungünstige prognostische Faktoren sowie mittlere Überlebenszeiten beim kutanen Lymphom des Hundes [10].

\begin{tabular}{|l|l|}
\hline prognostisch günstig & prognostisch ungünstig \\
\hline $\begin{array}{l}\text { solitäre Läsion: } \\
\text { mittlere Überlebenszeit 231 Tage }\end{array}$ & $\begin{array}{l}\text { multiple Läsionen: } \\
\text { mittlere Überlebenszeit 104 Tage }\end{array}$ \\
\hline $\begin{array}{l}\text { mukokutane oder orale Läsionen: } \\
\text { mittlere Überlebenszeit 491 Tage }\end{array}$ & $\begin{array}{l}\text { kutane Läsionen: } \\
\text { mittlere Überlebenszeit 103 Tage }\end{array}$ \\
\hline initial komplette Remission durch Zytostatika & partielle Remission oder kein Ansprechen auf Zytostatika \\
\hline
\end{tabular}

kann aufgrund der Radiosensibilität von Lymphoblasten auch eine Bestrahlung in Betracht gezogen werden [11]. Für die Therapie von multiplen oder generalisierten Hautveränderungen sollte man eine systemische Therapie empfehlen. Hierfür stehen zahlreiche Optionen zur Verfügung ( $\triangleright$ Tab.2). Die Vielzahl der Protokolle zeigt jedoch auch auf, dass bis heute kein Goldstandard der Therapie mit sehr guten Ergebnissen existiert.

Auch wenn die Krankheit nicht geheilt werden kann, ist das primäre Ziel der Therapie, die Lebensqualität der Patienten zu verbessern. Hierbei spielt auch die Kontrolle von Juckreiz und Sekundärinfektionen eine wichtige Rolle.

Aktuell liefern Studien mit der Behandlung durch das Chemotherapeutikum Lomustin die vielversprechendsten Ergebnisse [12]. Da es sich um eine orale Supplementation alle 3 Wochen handelt, ist der organisatorische Aufwand für die Besitzer vergleichsweise gering. An Tag 7 und 21 der Behandlung sollte eine Kontrolle von Blutbild und den Leberenzymaktivitäten erfolgen, da als Nebenwirkungen vor allem eine Suppression des Knochenmarks sowie ein Anstieg der Leberwerte beschrieben sind [13]. Die Ansprechrate ist mit $78-82 \%$ recht hoch $[14,15]$. Trotzdem beträgt die mittlere Überlebensrate lediglich 6 Monate. Des Weiteren gibt es zahlreiche andere intravenöse Chemotherapieprotokolle. Aufgrund des höheren Nebenwirkungspotenzials, den höheren Kosten und dem höheren Aufwand der Gabe sind diese aktuell der Behandlung mit Lomustin nicht überlegen.

Als alternative Behandlungsoption hat sich in den letzten Jahren außerdem die Gabe von Tyrosinkinase-Inhibitoren (z.B. Masitinib) bewährt. Der Wirkmechanismus beruht wahrscheinlich auf einem antiproliferativen Effekt auf die lymphoiden Zellen. In einer Studie konnte in keiner der Proben der c-KIT-Rezeptor nachgewiesen werden [16]. 
Wenn lediglich eine palliative Behandlung infrage kommt, kann die Gabe von Kortikosteroiden erwogen werden. In wenigen älteren Studien ist außerdem die Behandlung mit ungesättigten Fettsäuren oder Vitamin-APräparaten beschrieben $[17,18]$. Die Ansprechrate erscheint zunächst gut, jedoch fehlen größere Studien mit hohen Fallzahlen.

\section{Prognose}

Die Überlebenszeit der Patienten ist sehr variabel und schwankt zwischen wenigen Monaten bis zu 2 Jahren. Ein Großteil der Hunde überlebt jedoch das 1. Jahr nach der Diagnosestellung nicht. Ohne Behandlung beträgt die mittlere Überlebenszeit ca. 50 Tage. Eine Behandlung ausschließlich mit Glukokortikoiden verlängert diesen Zeitraum nur unwesentlich auf ca. 60 Tage [10]. In - Tab. 3 sind Faktoren gelistet, die sich günstig oder ungünstig auf die Prognose auswirken. Die Euthanasie stellt die häufigste Todesursache dar. Hierfür kann eine Verschlechterung des Hautbilds oder eine Generalisierung des Lymphoms verantwortlich sein.

\section{Fazit}

Das kutane Lymphom ist eine seltene tumoröse Erkrankung des Hundes, die durch die variablen Erscheinungsformen eine diagnostische Herausforderung für den Tierarzt darstellt. Vor allem bei älteren Tieren mit unklaren Hautsymptomen sollte deshalb zeitnah eine Biopsieentnahme erwogen werden. Es stehen nach der Diagnosestellung zahlreiche Therapieoptionen zur Verfügung. Die Prognose ist insbesondere bei generalisierter Ausprägung schlecht.

\section{Korrespondenzadresse}

Tierärztin Tanja Töpfer
Klinik für Kleintiere
Universität Leipzig
An den Tierkliniken 23
04103 Leipzig
tanja.toepfer@kleintierklinik.uni-leipzig.de

Literatur

[1] Fontaine J, Bovens C, Betteany S et al. Canine cutaneous epitheliotropic T-cell lymphoma: a review. Vet Comp Oncol 2009; 7 (1): 1-14

[2] Miller WH, Griffin CE, Campbell KL. Muller and Kirk's Small Animal Dermatology. $7^{\text {th }}$ ed. St. Louis, Missouri: Elsevier Saunders; 2012

[3] Santoro D, Marsella R, Hernandez J. Investigation on the association between atopic dermatitis and the development of mycosis fungoides in dogs: a retrospective case-control study. Vet Dermatol 2007; 18 (2): 101-106
[4] Kim E], Hess S, Richardson SK et al. Immunopathogenesis and therapy of cutaneous T cell lymphoma. J Clin Invest 2005; 115 (4): 798-812

[5] Thrall MA, Macy DW, Snyder SP et al. Cutaneous lymphosarcoma and leukemia in a dog resembling Sézary syndrome in man. Vet Pathol 1984; 21 (2): 182-186

[6] Fontaine J, Heimann M, Day M]. Canine cutaneous epitheliotropic T-cell lymphoma: a review of 30 cases. Vet Dermatol 2010; 21 (3): 267-275

[7] Fernandez NJ, West KH, Jackson ML et al. Immunohistochemical and histochemical stains for differentiating canine cutaneous round cell tumors. Vet Pathol 2005; 42 (4): 437-445

[8] Chaubert P, Baur Chaubert AS, Sattler U et al. Improved polymerase chain reaction-based method to detect early-stage epitheliotropic T-cell lymphoma (mycosis fungoides) in formalin-fixed, paraffin-embedded skin biopsy specimens of the dog. J Vet Diagn Invest 2010; 22 (1): 20-29

[9] Moore PF, Affolter VK, Keller SM. Canine inflamed nonepitheliotropic cutaneous T-cell lymphoma: a diagnostic conundrum. Vet Dermatol 2013; 24 (1): 204-211

[10] Chan CM, Frimberger AE, Moore AS. Clinical outcome and prognosis of dogs with histopathological features consistent with epitheliotropic lymphoma: a retrospective study of 148 cases (2003-2015). Vet Dermatol 2018; 29 (2): 154-159

[11] Santoro D, Kubicek L, LU B et al. Total skin electron therapy as treatment for epitheliotropic lymphoma in a dog. Vet Dermatol 2017; 28 (2): 246-265

[12] Laprais A, Olivry T. Is CCNU (lomustine) valuable for treatment of cutaneous epitheliotropic lymphoma in dogs? A critically appraised topic. BMC Vet Res 2017; 13 (1): 61

[13] Heading KL, Brockley LK, Bennett PF. CCNU (lomustine) toxicity in dogs: a retrospective study (2002-07). Aust Vet J 2011; 89 (4): 109-116

[14] Risbon RE, de Lorimier LP, Skorupski K et al. Response of canine cutaneous epitheliotropic lymphoma to lomustine (CCNU): a retrospective study of 46 cases (1999-2004). J Vet Intern Med 2006; 20 (6): 1389-1397

[15] Williams LE, Rassnick KW, Power HT et al. CCNU in the treatment of canine epitheliotropic lymphoma. J Vet Intern Med 2006; 20 (1): 136-143

[16] Holtermann N, Kiupel M, Kessler M et al. Masitinib monotherapy in canine epitheliotropic lymphoma. Vet Comp Oncol 2016; 14 Suppl 1: 127-135

[17] Iwamoto K, Bennett LR, Norman A et al. Linoleate produces remission in canine mycosis fungoides. Cancer Lett 1992; 64 (1): $17-22$

[18] White SD, Rosychuck RA, Scott KV et al. Use of isotretinoin and etretinate for the treatment of benign cutaneous neoplasia and cutaneous lymphoma in dogs. J Am Vet Med Assoc 1993; 202 (3): 387-391

Bibliografie

DOI https://doi.org/10.1055/a-0577-9115

kleintier konkret 2018; 21: 5-12

(c) Georg Thieme Verlag KG Stuttgart · New York ISSN 1434-9132 